\title{
Low density lipoprotein-induced lipid accumulation is a key phenomenon of atherogenesis at the arterial cell level
}

\author{
Alexander N. Orekhov, Veronika A. Myasoedova \\ Institute of General Pathology and Pathophysiology, Moscow 125315, Russia.
}

Correspondence to: Dr. Alexander N. Orekhov, Institute of General Pathology and Pathophysiology, Moscow 125315, Russia. E-mail:a.h.opexob@gmail.com

How to cite this article: Orekhov AN, Myasoedova VA. Low density lipoprotein-induced lipid accumulation is a key phenomenon of atherogenesis at the arterial cell level. Vessel Plus 2019;3:3. http://dx.doi.org/10.20517/2574-1209.2018.80

Received: 22 Dec 2018 First Decision: 24 Dec 2018 Revised: 2 Jan 2019 Accepted: 3 Jan 2019 Published: 13 Feb 2019

Science Editor: Alexander N. Orekhov Copy Editor: Cui Yu Production Editor: Huan-Liang Wu

\begin{abstract}
Lipid accumulation in cells of subendothelial intima and the formation of foam cells is the earliest and the most noticeable manifestation of atherosclerosis at the cellular level. Generally, the foam cell formation is the result of interaction of cell with pro-atherogenic low-density lipoprotein providing cholesterol delivery and anti-atherogenic highdensity lipoprotein providing its efflux. In this review, we discuss possible mechanisms of foam cell formation, the role of intracellular lipid deposition as a trigger of atherosclerotic lesion development, current approaches to diagnostics and strategies for preventing atherosclerosis based on recent knowledge of causes of foam cell formation.
\end{abstract}

Keywords: Atherogenesis, atherosclerosis, foam cells, diagnostics, desialylation, low-density lipoprotein, subendothelial cells, therapy

\section{INTRODUCTION}

In most cardiovascular diseases epidemiology is related to atherosclerosis. As early as atherosclerotic lesions began to be studied under a microscope, it was found that the fundamental difference of atherosclerotic cells from normal ones is their foamy cytoplasm. This foamy structure is explained by numerous lipid inclusions in the form of fatty drops and granules that often fill the whole cell body and its processes. Lipid accumulation in cells of subendothelial intima and the formation of foam cells is the earliest and the most noticeable manifestation of atherosclerosis at the cellular level. At any stage, from the earliest microscopic

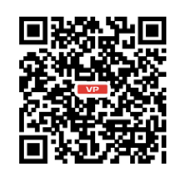


changes to the advanced plaque, atherosclerotic lesion is characterized by the deposition of intracellular lipids (mainly cholesteryl esters) in arterial sub-endothelial cells ${ }^{[1,2]}$.

Generally, the foam cell formation is the result of interaction of cells with pro-atherogenic low-density lipoprotein (LDL) providing cholesterol delivery and anti-atherogenic high-density lipoprotein (HDL) providing its efflux. Foam cell formation through the very LDL receptor by remnant lipoprotein and lipoprotein $(a)^{[3]}$ and through other ways has also been described. However, the main source of fat in the foam cells is plasma $\mathrm{LDL}^{[4]}$. In this review, we discuss possible mechanisms of foam cell formation and the role of intracellular lipid deposition as a trigger of atherosclerotic lesion development. In addition, the objective of the review is to discuss current approaches to preventing the accumulation of lipids in arterial cells because there is a general consensus that inhibition of foam cell formation is an important strategy for preventing atherosclerosis.

\section{MECHANISMS OF FOAM CELL FORMATION}

The obvious close connection of atherosclerosis with foam cells led to the idea that lipid accumulation is a necessary condition or fundamental even of atherogenesis. The origin of foam cells is still a matter of controversy. Currently, the dominant view is that foam cells have an exclusively macrophage origin. However, in the sub-endothelial intima of human arteries, macrophages represent a minority in comparison with smooth muscle $\alpha$-actin-positive cells (SMA+) that are typical smooth muscle cells and pericytes, however, even in the initial lesions, almost all cells contain lipid inclusions ${ }^{[5]}$. Consequently, not all cells loaded with lipids are macrophages. According to the most recent data, in human coronary lesions, the proportion of smooth muscle cells is higher than $50 \%$ of total number of foam cells ${ }^{[6]}$. Nevertheless, the culture of macrophages is widely used to study the mechanisms of foam cell formation, since the macrophage is a simple and convenient model. Taking into account the possible role of LDL in the accumulation of intracellular cholesterol, many research groups have attempted to induce the increase of cholesterol level in cultured cells by incubation with lipoprotein.

\section{Modified LDL}

In hyperlipidemia at high LDL levels, fluid-phase pinocytosis induces foam cell formation ${ }^{[7,8]}$. However, generally native LDL does not cause lipid storage in cell cultures ${ }^{[5,9,10]}$. On the other hand, considerable lipid accumulation was detected in the case of chemically modified LDL (acetylated, maleylated, succinylated, oxidized, etc.) or treatment with formaldehyde, malondialdehyde, phospholipase A2, C and D, etc. ${ }^{[10,11]}$. Therefore, chemically modified LDL is able to trigger foam cell formation, hence it is atherogenic. This discovery initiated the search for a naturally occurring atherogenic modified LDL. Proceeding from the hypothesis of oxidative modification, it was the oxidized LDL that was searched for but it was not found in the blood, although some indirect signs of oxidation of circulating LDL were detected by detecting a marker for evaluating the LDL oxidation in vivo ${ }^{[9,10]}$. A more reasonable way to detect the in vivo modified LDL, i.e., LDL particles capable of triggering lipid accumulation in cultured cells, was to search for such an atherogenic lipoprotein circulating in patients with atherosclerosis. In addition to oxidation, other forms of atherogenic modified LDL were discovered in the blood. Different groups at different times have found small dense LDL (sdLDL), electronegative or LDL(-), and desialylated lipoprotein particles circulating in the blood ${ }^{[9,10]}$.

It was found that LDL(-), sdLDL and desialylated LDL particles have many common characteristics and can all be oxidized ${ }^{[5,9,10]}$ [Table 1]. This led to the idea that all known forms of atherogenic modification of LDL can be present in the same lipoprotein particle, that is, there is multiple modification of LDL. In the blood, the same multiply modified LDL particles possess the properties of small dense, electro-negative, oxidized and desialylated lipoproteins ${ }^{[5,9,10]}$.

A mechanism of LDL multiple modification was proposed [Figure 1]. The hypothesis was tested under conditions imitating the situation in vivo. Native (unmodified) LDL particles were isolated from circulation 


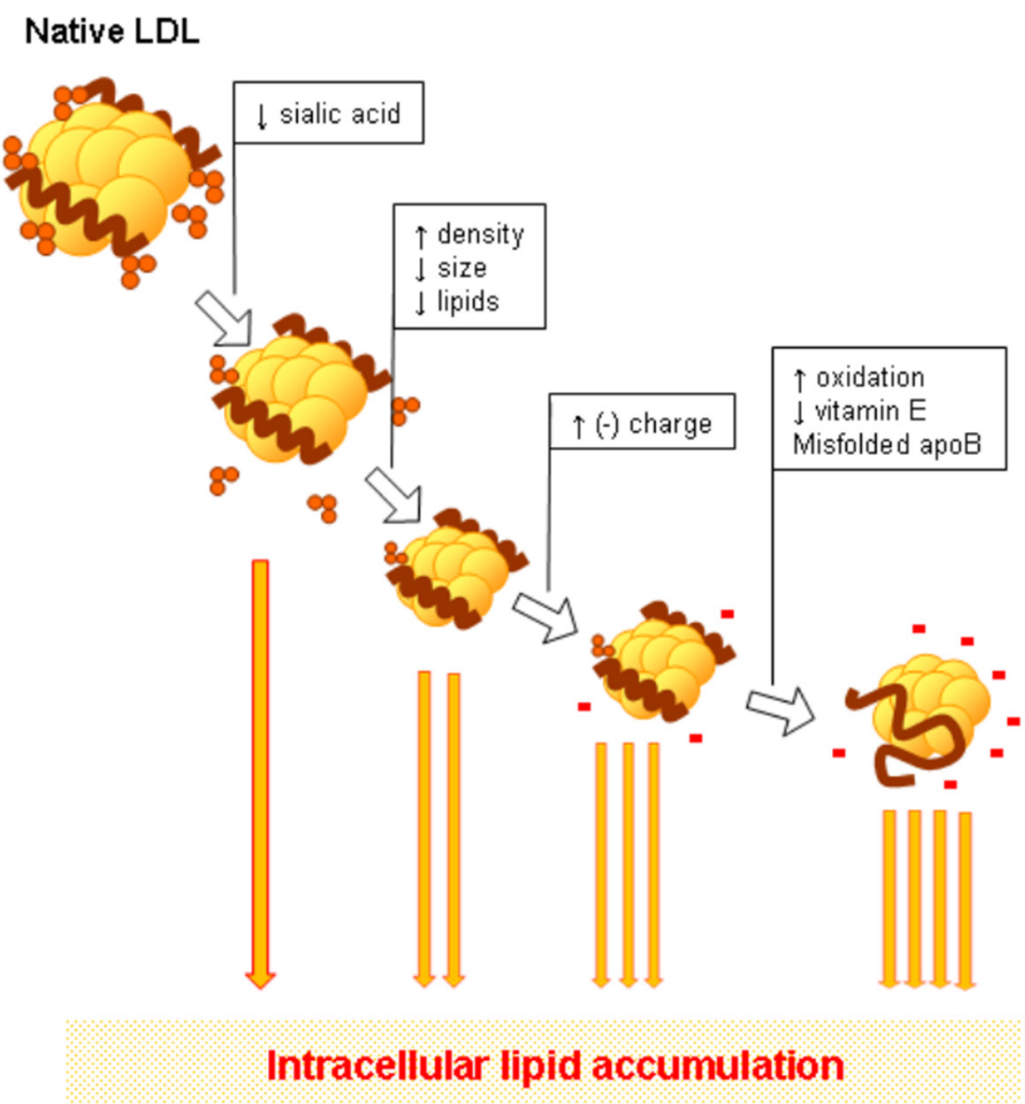

Figure 1. Cascade of multiple atherogenic modifications of low-density lipoprotein (LDL) particles. Adopted from ${ }^{[9]}$, with permission

of healthy subjects; and the serum was prepared from the blood of patient with atherosclerosis. Then the mixture of LDL and serum was incubated at $37^{\circ} \mathrm{C}$ during $72 \mathrm{~h}$.

After $1 \mathrm{~h}$ of incubation, sialic acid content of LDL decreased, and desialylated LDL particles appeared. LDL acquired the ability to induce accumulation of intracellular cholesterol; hence the lipoprotein particles became atherogenic. After 6 h, lipid content and LDL size were decreased. After 36 h, LDL became more electronegative. The incubation for 48-72 h caused a decrease in the content of antioxidants; in parallel, susceptibility of LDL to oxidation was increased, leading to the oxidation of the lipoprotein particles. Prolonged incubation led to degradation of apoproteins. The experiments described above allow us to conclude that desialylation is the first known modification that leads to the acquisition of atherogenic properties by LDL particles. Subsequent modifications, such as decrease of the lipid content and particle size, increase of the particle density and electronegativity further enhanced the LDL atherogenicity. Oxidative modification of lipoprotein particles occurs at the last stages of a cascade of events of sequential multiple modifications.

Despite the fact that LDL modification involves changes in different physical and chemical properties in lipoprotein particle, oxidation is still considered to be the main or even the only atherogenic modification. Currently, PubMed lists 9,765 publications indexed under "oxidized LDL", and 4,728 under "oxidized LDL and atherosclerosis". More than a thousand review articles on oxidized LDL have been published. It is generally accepted that oxidized LDL accumulating in the vascular wall triggers atherogenesis ${ }^{[5,9,10]}$. Although oxidized LDL was detected in the vascular wall ${ }^{[3]}$, for a long time it could not be detected in the bloodstream. This gave rise to the idea that LDL oxidation does not occur in the blood but takes place in the vascular wall. There is no need for such an assumption since, as mentioned above, a marker for evaluation 
Table 1. Characteristics of naturally occurring modified low-density lipoprotein discovered in circulation of atherosclerotic patients

\begin{tabular}{lccc}
\hline Parameter & sdLDL & LDL(-) & Desialylated LDL \\
\hline Intracellular cholesterol accumulation (atherogenicity) & $\uparrow$ & $\uparrow$ & $\uparrow$ \\
Size & $\downarrow$ & $\downarrow$ & $\downarrow$ \\
Density & $\uparrow$ & $\uparrow$ & $\uparrow$ \\
(-) Charge & $\uparrow$ & $\uparrow$ & $\uparrow$ \\
Sialic acid & $\downarrow$ & $\downarrow$ & $\downarrow$ \\
Cholesteryl esters & $\downarrow$ & $\downarrow$ & $\downarrow$ \\
Phospholipids & $\uparrow$ & $\downarrow$ & $\downarrow$ \\
Protein/lipids & $\uparrow$ & $\uparrow$ & $\uparrow$ \\
Oxidizability & $\uparrow$ & $\uparrow$ & $\uparrow$ \\
Oxidation & $\uparrow$ & $\uparrow$ & $\uparrow$ \\
Antioxidants & $?$ & $\uparrow$ & $\uparrow$ \\
Amino group modification & $\uparrow$ & $\uparrow$ & $\uparrow$ \\
Self-association & $\uparrow$ & $\uparrow$ \\
\hline
\end{tabular}

Adopted from ${ }^{[9]}$, with permission. LDL: low-density lipoprotein; sdLDL: small dense LDL; $\uparrow:$ increased; $\downarrow$ : decreased; ?: not known

of LDL oxidation was detected in the blood of atherosclerotic patients ${ }^{[5,9,10]}$. Earlier, some signs of oxidation were found in LDL isolated from the blood ${ }^{[5,9,10]}$.

The following arguments are usually made in favor of the fact that oxidized LDL plays a key role in atherogenesis: (1) in the blood of patients, antibodies to malondialdehyde-LDL (MDA-LDL) were discovered $^{[5,9,10]}$; (2) antibodies obtained to in vitro oxidized LDL co-localized with LDL in histological sections of atherosclerotic lesions ${ }^{[5,9,10]}$; and (3) LDL isolated from the artery wall possessed some characteristic properties of oxidized $\mathrm{LDL}^{[12]}$. However, all these arguments can be questioned. First, MDALDL cannot be regarded as an adequate model of oxidized LDL. Usually, antibodies are produced against LDL modified in vitro with MDA or copper-induced oxidation. This modification leads to random and non-specific exposure of immunogenic sites different from those present in LDL oxidized in vivo. Second, antibodies isolated from the blood show cross-reactivity with MDA-LDL, naturally occurring multiply modified LDL and in vitro desialylated LDL. Anti-LDL antibodies possess the maximal affinity for desialylated LDL; the affinity for oxidized LDL is an order of magnitude lower ${ }^{[5,9,10]}$. Thus, antibodies are produced primarily to the desialylated LDL. Third, although oxidized LDL is not detected in the blood, other forms of atherogenic modification (sdLDL, LDL- and desialylated LDL) are clearly detected in the circulating lipoprotein. Multiply modified atherogenic LDL circulating in the blood of atherosclerotic patients was detected, isolated and intensively studied ${ }^{[5,9,10]}$. The presence of sdLDL in the blood is currently known and well-studied providing some impact on the anti-atherosclerotic therapy ${ }^{[5,9,10]}$. The results of intensive study of LDL- have found implications in both diagnostics and therapy ${ }^{[5,9,10]}$. Despite numerous attempts of clinical implementation of methods for determining MDA-LDL, they are still not widely used. Fourth, as mentioned above, modification of LDL in the plasma of atherosclerotic patients begins with desialylation followed by changes in lipid content, decrease in size and increase in density. At later stages, LDL becomes more electronegative. Only at the very end of this cascade of transformations, the lipoprotein acquires the properties of oxidized particle [Figure 1]. Thus, oxidation is neither the primary nor the only atherogenic modification of LDL.

Taking into account the key role of the multiply modified atherogenic LDL in the initiation and progression of atherosclerosis, it is reasonable to assume that atherosclerotic risks are dependent not as much on the total content of LDL in the blood as on the level of multiply modified LDL. In this regard, the level of multiply modified LDL should be a better biomarker of atherosclerosis than the total LDL level. Such a conclusion will be true for HDL because dysfunctional HDL has been detected in the blood of atherosclerotic patients.

To understand which modification plays the key role conveying atherogenic properties of a lipoprotein particle, we studied correlations between the changes in chemical and physical parameters of the multiply 
modified LDL and its ability to induce intracellular cholesterol accumulation. The only statistically significant correlation was found between the atherogenic potential of LDL and sialic acid content of the particles. This correlation was reversed: the lower was the sialic acid content of LDL, the more cholesterol was accumulated by the cells. LDL parameters such as size, charge, lysine-free amino groups, phospholipids and neutral lipids content, fat-soluble antioxidants, lipid peroxidation products, oxidation and oxidation rates did not correlate significantly with LDL atherogenicity ${ }^{[5,9,10]}$. Thus, desialylation appeared to be the most important modification inducing LDL atherogenicity. Presumably, the necessary and sufficient condition for the appearance of atherogenic properties in LDL particles is desialylation.

Studying of the mechanisms of LDL desialylation led to the discovery of trans-sialidase activity in the blood of atherosclerotic patients ${ }^{[5,9,10]}$. It was present both in association with lipoproteins and in free form in blood plasma. The exact identity of the sialidase activity is not known, yet, however, LDL treated with isolated trans-sialidase lost sialic acid and became atherogenic causing the accumulation of cholesteryl esters in cultured cells ${ }^{[5,9,10]}$. Thus, trans-sialidase found in the blood causes LDL-lipid desialylation, which leads to foam cells formation. Circulating trans-sialidase is a protein of about $65 \mathrm{kDa}$, whose content ranges from 20-200 $\mu \mathrm{g} / \mathrm{mL}$. Three optimum $\mathrm{pH}$ values were found: 3.0, 5.0 and 7.0. The enzyme activity was found to be stimulated by calcium and magnesium ions and dependent on the sulfhydryl groups. Donors of sialic acid for trans-sialidase are LDL, intermediate-density lipoprotein (IDL), very low-density lipoprotein (VLDL), and HDL. Preferable substrate is LDL, but VLDL, IDL and the least HDL may also serve as substrate. It is likely that trans-sialidase plays a key role in the in vivo atherogenic modification of lipoprotein particles.

\section{LDL association}

The formation of different associates containing modified LDL considerably increase lipoprotein atherogenicity. There were found at least three ways of potentiating LDL atherogenicity: self-association of LDL particles, LDL association with the extracellular matrix components and formation of LDL-containing immune complexes.

Unlike native LDL, desialylated LDL particles are associated with spontaneously in vitro in cell culture conditions $^{[5,9,10]}$. A significant direct correlation was found between the atherogenicity of desialylated LDL and the degree of lipoprotein particles' association ${ }^{[5,9,10]}$. If the LDL associates formed in culture are removed from the medium by filtration, this completely prevents the intracellular cholesterol accumulation. It was shown that increased atherogenicity of desialylated LDL self-associates is a result of increased uptake of lipoprotein particles by phagocytosis and a decrease in the rate of intracellular degradation ${ }^{[5,9,10]}$. If the selfassociates of LDL circulate in the blood of atherosclerotic patients or are formed in the arterial intima, then self-association is the actual mechanism of increasing the atherogenic potential of desialylated LDL.

Another mechanism of enhancing atherogenicity of desialylated LDL, namely, the formation of lipoprotein associates with the connective tissue matrix components has been found ${ }^{[11]}$. It was reported that LDL forms associates with collagenase-resistant arterial debris, collagen, elastin and proteoglycans isolated from human aortic intima ${ }^{[5,9,10]}$. All these associates caused cholesterol accumulation in cultured cells. On the other hand, LDL(-) does not induce the formation of foam cells but high susceptibility of LDL(-) to association is a factor that favors the formation of foam cells ${ }^{[13]}$.

Desialylation of LDL particles provokes the production of autoantibodies that form circulating immune complexes (CIC) containing LDL. Such CIC were detected in the blood of atherosclerotic patients ${ }^{[5,9,10]}$. A correlation was found between the levels of LDL containing immune complexes in the blood serum and the severity of atherosclerosis in patients ${ }^{[5,9,10]}$. It was found that LDL isolated from CIC is not only desialylated, but is also small, dense, more electronegative, and has decreased the content of neutral lipids, phospholipids and neutral saccharides, and is also characterized by conformational changes in the tertiary apoB structure. These findings allow considering LDL from CIC to be identical to multiply-modified LDL. LDL-containing 
CIC potentiate the atherogenicity of desialylated LDL causing higher cholesterol accumulation in cultured cell $^{[5,9,10]}$. The addition of the complement component and fibronectin to the LDL autoantibody complex $\mathrm{C} 1 \mathrm{q}$ resulted in a more pronounced accumulation of intracellular cholesterol. Thus, the formation of CIC is the actual mechanism for enhancing the atherogenic capacity of desialylated LDL. In addition to the accumulation of lipids, the CIC induces the secretion of pro-inflammatory cytokines and apoptosis of macrophages ${ }^{[12]}$ which can also be considered as pro-atherogenic effect. Recent studies of LDL containing CIC focus mainly on implementation into clinical practice for diagnostics (see below).

\section{FOAM CELLS AND ATHEROGENESIS}

Intracellular lipid accumulation leading to foam cell formation is the most noticeable and earliest manifestation of atherosclerosis. However, other manifestations are also generally recognized: (1) an increase in the proliferative activity of the leader leading to hypercellularity; (2) the enhancement of synthesis of the extracellular matrix, leading to the growth of connective tissue; and (3) the loss of intercellular contacts leading to rupture of the cellular network in the intima and detachment of intimal cells ${ }^{[5,14]}$. Examined was the effect of circulating desialylated LDL on proliferative activity and synthesis of total protein, collagen and glycosaminoglycans by SMA+ cells cultured from uninvolved human aortic intima.

A $24 \mathrm{~h}$ incubation of SMA+ cells with native LDL had no effect on proliferative activity detected as $\left[{ }^{3} \mathrm{H}\right]$ thymidine incorporation into cultured cells ${ }^{[5]}$. By contrast, addition of desialylated LDL subfraction leads to a 1.5- to 2 -fold increase of proliferative activity.

The rate of synthesis of proteins secreted by cultured cells was evaluated by incorporation of $\left[{ }^{14} \mathrm{C}\right]$ leucine in the acid-insoluble fraction of culture medium. Native LDL had no effect on the synthesis of secreted proteins. Desialylated LDL stimulated protein syntheses by $1.5-$ to $2-$ fold $^{[5]}$. Moreover, desialylated LDL induced a 2 -fold increase of collagen production, as estimated by incorporation of $\left[{ }^{14} \mathrm{C}\right]$ proline in the collagenasereleased fraction of culture medium ${ }^{[5]}$. It was also demonstrated that desialylated LDL, but not native LDL, stimulates the incorporation of $\left[{ }^{14} \mathrm{C}\right]$ glucosamine in the total glycosaminoglycan fraction of human SMA+ cells $^{[5]}$. So, in contrast to native LDL, desialylated LDL enhanced synthesis of connective tissue matrix components.

It was shown that: (1) desialylated LDL-induced lipid accumulation is sufficient to enhance proliferative activity and stimulates the incorporation of precursors of the extracellular matrix components; (2) insoluble complexes of native LDL with naturally occurring (collagen, elastin, fibronectin) and artificial (latex particles, dextran sulfate) compounds induce intracellular lipid accumulation and stimulate proliferative and synthetic activities; and (3) the increase in proliferative and synthetic activities correlates with the amount of accumulated intracellular cholesterol ${ }^{[5]}$. In addition, after incubation of SMA+ cells with desialylated LDL, intercellular communication through gap junctions was dropped considerably ${ }^{[14]}$. These findings indicate that intracellular lipid accumulation might be a reason for the disintegration of cellular network observes in atherosclerotic lesions.

Thus, foam cell formation induced by desialylated LDL causes enhanced proliferative activity, the synthesis of the connective tissue matrix components and also breaks intercellular communication. Therefore, desialylated LDL can induce all known atherosclerotic manifestations at the cellular level.

The interaction of atherogenic modified LDL with macrophages causes a pro-inflammatory response which, if unfavorable, develops into a local chronic inflammation characteristic of atherosclerotic lesions. In primary human monocyte-derived macrophages, modified LDL-induced cholesterol accumulation is accompanied by upregulation of genes encoding pro-inflammatory molecules ${ }^{[15]}$. It is unclear whether foam cell formation induces a pro-inflammatory response or pro-inflammatory response promotes cholesterol accumulation. It was demonstrated that local cellular responses to oxidized LDL may stimulate pro- 
inflammatory or anti-inflammatory pathways ${ }^{[16]}$. Oxidized LDL loading of macrophages negatively regulates pro-inflammatory gene expression and implicates epigenetic mechanisms such as histone deacetylase activity $^{[17]}$. On the other hand, an increase in circulating levels of IL-17 has been demonstrated in patients with cardiovascular disease, as well as its high expression in atherosclerotic lesions, suggesting that IL-17 could affect cell targets such as macrophages in atherosclerotic lesion. In this sense, it has been shown that IL-17 alone induces few foam cells ${ }^{[18]}$.

\section{FACTORS AFFECTING THE FORMATION OF FOAM CELLS}

Once the ability of atherogenic modified LDL circulating in the blood of atherosclerotic patients to cause lipid accumulation in cultured cells (atherogenicity or atherogenic potential) has been found, a natural desire to check agents that potentially could influence the foam cell formation arose. Interest was motivated by the fact that the detection of an agent preventing the accumulation of intracellular lipids could be considered as the discovery of a potential anti-atherogenic drug. Hundreds of different substances were tested. Among them, those that suppressed the accumulation of lipids in cultured cells, and those that increased the atherogenic potential of LDL were detected; there were also such agents that had no effect ${ }^{[19]}$.

An important part of the most recent works devoted to foam cell formation is associated precisely with the search for modulators of intracellular lipid accumulation. Ceramide generated as a result of aggregated LDL catabolism in atherosclerotic plaques activates macrophage $\mathrm{RhoA}^{[20]}$. RhoA activation plays a significant role in macrophage RhoA/Rho kinase signaling that decreases aggregated LDL degradation and foam cell formation by reducing local actin polymerization required for catabolism. This may be regarded as a possible anti-atherosclerotic effect. Proline/serine-rich coiled-coil 1 overexpression reduced foam cell formation trough decrease of intracellular cholesterol and increase of cholesterol efflux by upregulating the expression of peroxisome proliferator-activated receptor $\gamma$ and liver X receptor $\alpha^{[21]}$.

A flavonoid from the Morus alba L., Kuwanon G, inhibits both cholesterol accumulation and inflammation reaction in macrophages stimulated by oxidized $\mathrm{LDL}^{[22]}$. Observed effects are realized through enhancing LXR $\alpha-A B C A 1 / A B C G 1$ pathway and inhibiting NFאB activation. Anti-atherosclerotic effect of Kuwanon G was confirmed in vivo. In the plaque of high-fat diet fed ApoE -/- mice, Kuwanon G significantly reduces the atherosclerotic areas and macrophage content. Nuclear factor erythroid 2-related factor 2 (Nrf2) prevents foam cells formation ${ }^{[23]}$. On the other hand, the loss of Nrf2 in macrophages enhances foam cell formation and promotes early atherogenesis. Isolated from the root of Salvia miltiorrhiza Bge., Tanshindiol C, an activator of $\mathrm{Nrf} 2$ in macrophages, markedly suppresses foam cell formation induced by oxidized LDL ${ }^{[24]}$. Liver kinase B1 suppresses foam cell formation and atherosclerosis development. On the contrary, downregulation of liver kinase B1 in macrophages results in such atherogenic manifestations as increased uptake of modified lipoproteins, increased foam cell formation, and, finally, increased atherosclerosis ${ }^{[25]}$.

Antibodies to specific epitopes of oxidized LDL suppress both inflammatory cytokine production and foam cell formation ${ }^{[26,27]}$.

Endogenous human vascular endothllial growth factor (VEGF) inhibits foam cell formation. VEGF-treated macrophages significantly decreased lipid accumulation caused by oxidized trough down-regulation of CD36 expression ${ }^{[28]}$.

The studies of agents that promote foam cell formation provide novel insights into their pro-atherogenic effects under pathological conditions and suggests that their inhibiting may represent a new approach for treating atherosclerosis. The following is a couple of examples of such studies. Hypoxia-inducible lipid droplet-associated protein was shown to be highly expressed in atherosclerotic foam cells in human and 
murine plaques. It was established that lipid droplet proteins can promote atherogenesis being critical to the foam cell formation and lipid deposition ${ }^{[29]}$. Lysophosphatidic acid (LPA) is a bioactive phospholipid produced by activated platelets that is formed during the oxidation of LDL. The formation of foam cells is significantly enhanced by LPA alone through upsetting the imbalance between lipid uptake and efflux ${ }^{[30]}$.

\section{CLINICAL APPLICATIONS}

The results obtained in clinical studies did not always correspond to the theoretical understanding of the role of LDL in atherogenesis. As follows from the concept of evidence-based medicine, the mechanistic role of biomarkers should be confirmed by clinical studies (trials and surveys) ${ }^{[31]}$. In other words, the modulation of the biomarker should affect the established endpoints. In the case of atherosclerotic diseases, such endpoints are: fatal and non-fatal cardiovascular events, angina pectoris, revascularization, fatal and nonfatal myocardial infarction, stroke, etc. When talking about the direct relationship with atherosclerosis, endpoints often use surrogate instrumental methods, namely angiography of coronary arteries, ultrasound imaging of the carotid intima-media thickness and, less commonly, calcification of the coronary arteries.

\section{Diagnostics}

Unfortunately, modified LDL is not used in American and European recommendations and guidelines for reducing the risk of developing atherosclerotic cardiovascular diseases ${ }^{[32-34]}$. This is the result of the fact that the available data from clinical studies do not allow us to justify the use of modified LDL and HDL as mechanistic biomarkers of atherosclerotic disease. Apparently, the results of such studies would be more encouraging if atherogenic multiply modified LDL and dysfunctional HDL were considered as pharmacological targets and biomarkers.

The difficulties in verifying new biomarkers are related to the complexity of interaction of different markers and risk factors. In addition, against the background of usual risk factors, new biomarkers are often not supported by evidence of their ability to contribute to the assessment of atherosclerotic risk and possess no significant diagnostic or prognostic role ${ }^{[35-38]}$. Currently, numerous traditional and experimental biomarkers are considered to assess atherosclerotic risk. Along with lipid markers, which include modified LDL, dysfunctional HDL and apolipoproteins, non-lipid markers are considered. Non-lipid biomarkers include inflammatory molecules, namely fibrinogen and a highly sensitive C-reactive protein. Lipoprotein-associated phospholipase A2 and homocysteine are considered as thrombotic markers. Other indexes are glucose metabolism markers and organ-specific markers.

Clinical studies of modified LDL as a biomarker of atherosclerosis have certain limitations. In particular, the use of the term "oxidized LDL" causes confusion. To measure the allegedly "oxidized" LDL in clinical trials, antibodies against MDA-LDL are used. Such an indicator cannot be called oxidized LDL. It is clear that in this case it is possible to evaluate the clinical significance of some indicator whose physical meaning is difficult to interpret. Autoantibodies against LDL found in the blood of atherosclerotic patients cross-react with MDA-LDL, multitude modified LDL and desialylated $\mathrm{LDL}^{[39]}$. Moreover, these autoantibodies possess the highest affinity with desialylated LDL while they did not distinguish between native and $\mathrm{Cu}^{2+}$-oxidized LDL. Therefore, MDA-LDL to some extent evaluate the desialylated and not oxidized LDL.

Since it was impossible to directly demonstrate the presence of oxidized LDL in the bloodstream, the term "minimally oxidized LDL" has been introduced. This should imply some hypothetical particle that cannot be isolated or characterized by existing methods, but which actually exists penetrating the artery wall, undergoing further modification, and triggering atherosclerosis ${ }^{[40]}$. Unfortunately, such "pure faith" is widespread and is not subject to revision.

As a result of experimental or clinical studies of oxidized LDL, both indirect and direct oxidation indices can be obtained. Indirect indices are based on the measurements using anti-MDA-LDL or anti-copper- 
oxidized LDL-antibodies. The limitations of these approaches were discussed above. Direct markers of oxidation are based on evaluating oxidized lipids including phospholipids. A non-pathological retard in LDL catabolism which is reflected in the appearance of senescent LDL in the blood may be a limitation of this approach. It should be noted that the clinical studies did not examine the mechanistic significance of the modified LDL in atherogenesis but tried to position it as a biomarker. Admittedly, these attempts were unsuccessful and did not lead to definitive conclusions about the sensitivity and specificity of modified LDL.

LDL(-) as a diagnostic parameter attracts the attention of several groups ${ }^{[41]}$. There are certain successes in the implementation of this indicator in clinical practice ${ }^{[42]}$.

The ability of atherogenic modified LDL to cause the accumulation of cholesterol in cultured cells could be used to create a diagnostic test system for assessing the atherogenicity of lipoproteins. However, the cellular test is not a useful biomarker for implementation in clinical practice. A search for more adequate tests was conducted. It was found that the LDL level in the CIC very closely correlates with the value of LDL atherogenicity estimated in the cellular test ${ }^{[43-45]}$. The most successful were attempts to introduce into the clinic such a parameter as the content of LDL in the CIC. The association between progression of cardiovascular diseases and high levels of cholesterol in precipitated CIC has been found ${ }^{[46,47]}$. The high diagnostic and prognostic significance of this parameter was also demonstrated for carotid and coronary atherosclerosis ${ }^{[43-45,48,49]}$.

\section{Therapy}

The mechanistic role of oxidized LDL as an effector of atherosclerosis could be confirmed by clinical trials using antioxidants. To the complete disappointment of supporters of the oxidative theory, randomized trials have shown a lack of risk reduction or even an increased risk of atherosclerosis in patients receiving antioxidants ${ }^{[50]}$. Despite attempts to explain the negative results, the topic of oxidized LDL in atherosclerosis was closed. Unfortunately, along with this, the interest to general problems of modified LDL was lost at all. As a result, the American Heart Association did not recommend antioxidants for the prevention of atherosclerotic diseases ${ }^{[51]}$ although the question on modified LDL as a mechanistic factor or biomarker of atherosclerosis remains open.

Over the past two decades, data demonstrating the non-linear dependence of the reduction in the risk of atherosclerosis from the therapeutic reduction in total LDL level are widely discussed ${ }^{[52-61]}$. Figure 2 clearly shows that the clinical manifestations of atherosclerosis are due not only to the total level of LDL in the blood. It is necessary to conduct similar studies with measuring the level of modified LDL in order to evaluate its value as a mechanistic effector.

In contrast to the outdated concepts of the total level of circulating LDL as a cause for the formation of atherosclerotic lesion, it seems more reasonable the idea that the key initiating event in atherogenesis is the retention, or trapping, of LDL within the arterial wall ${ }^{[62,63]}$. The paradigm of the key role of LDL and foam cells in atherogenesis gave rise to the concept of cellular cholesterol retention ${ }^{[64,65]}$. Cell models have been developed to evaluate the effect of various agents on the retention of cellular cholesterol caused by atherogenic modified $\mathrm{LDL}^{[66]}$.

In the in vitro model, primary cultures of human aortic cells or human monocyte-derived macrophages are used for the screening of potential drugs, the investigation of their mechanisms of action, and the optimization of anti-atherosclerotic drug therapy. Cells of the subendothelial intima isolated from atherosclerotic lesions retain all major characteristics of atherosclerotic cells when cultured. Many cells cultured from atherosclerotic lesions are so-called foam cells, which contain numerous inclusions, likely lipid droplets, that fill the entirety of the cytoplasm ${ }^{[67]}$. The bulk of excess lipids in foam cells consists of free 


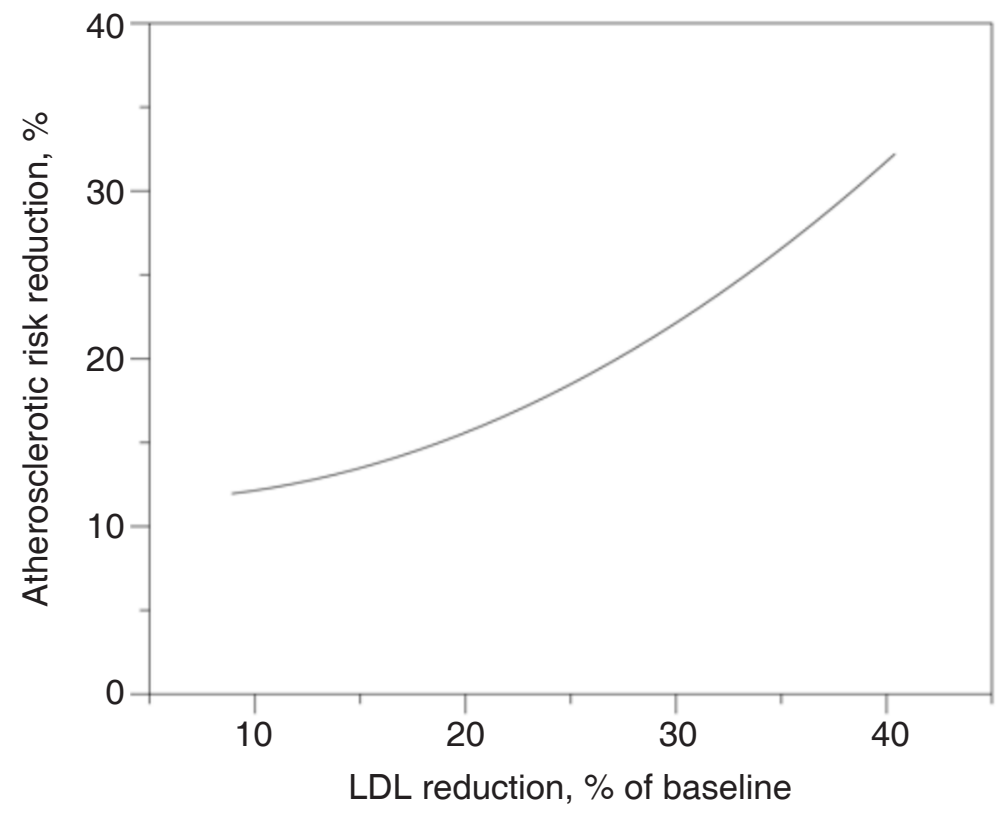

Figure 2. Consensus relationship between treatment effect on the low-density lipoprotein (LDL) level and the reduction in the risk of atherosclerosis-related cardiovascular events. Adopted from ${ }^{[73]}$, with permission

cholesterol and cholesteryl esters ${ }^{[67]}$. It should be noted that the content and composition of lipids in cultured cells within the first 10-12 days in culture remain unchanged and correspond to the respective indices of freshly isolated cells ${ }^{[67,68]}$.

In the ex vivo model, instead of drugs, blood sera taken from patients after oral drug administration is added to cultured cells. Cell cultures can be employed in an ex vivo model to examine an indirect anti-atherogenic action of a drug and to optimize anti-atherosclerotic (anti-atherogenic) drug therapies. The cellular models can be used not only to test drugs but can also be used to test foodstuffs as well. Anti-atherosclerotic (therapeutic, causing regression of atherosclerosis) and anti-atherogenic (preventive) activities of many agents were investigated. This approach will be useful in the development and optimization of anti-aterhosclerotic and anti-atherogenic dietary therapies. The anti-atherogenic effects of dietary products promote the development of anti-atherosclerotic therapies based on natural products. Atherosclerosis develops over many years, so anti-atherosclerotic therapies should be long-term or even lifelong. For such long-term therapies, conventional medicine will not work. Drugs based on natural products can be a good alternative. Among the anti-atherogenic natural products, the most effective was garlic. Two-year treatment with Allicor (garlic powder) has a direct anti-atherosclerotic effect on carotid atherosclerosis in asymptomatic men ${ }^{[6]]}$. Inflaminat (calendula, elder and violet), which possesses anti-cytokine activity, caused the regression of carotid atherosclerosis in a 1-year treatment of asymptomatic men ${ }^{[70]}$. The phytoestrogen-rich drug Karinat (garlic powder, extract of grape seeds, green tea leaves, hop cones, $\beta$-carotene, $\alpha$-tocopherol and ascorbic acid) prevented the development of carotid atherosclerosis in postmenopausal women ${ }^{[7]]}$.

Although some herbal preparations are recommended by the FDA; unfortunately, natural products that possess anti-atherosclerotic therapeutic potential are not prescribed by medical practitioners as antiatherosclerotic agents. However, the potential of these substances allows us to consider them as mainline additional supplements or prescriptions ${ }^{[72]}$.

\section{CONCLUSION}

The accumulation of intracellular lipids leading to foam cell formation is induced by modified LDL. This is a fundamental event in the genesis of atherosclerotic lesions. It is the accumulation of intracellular lipids that 
triggers atherogenesis at the cellular level. This knowledge should lead to the development of fundamentally new approaches to the diagnosis of atherosclerotic diseases, but unfortunately extensive research in this direction is not carried out. On the other hand, new developments based on foam cells and LDL were translated into clinics as therapeutic tools. Using cell models and natural products, an approach has been developed to prevent the formation of foam cells. This is an example of how the results of basic research have been successfully translated into clinical practice. Drugs with direct anti-atherosclerotic activity have been developed. These drugs cause regression of atherosclerosis and/or prevention of its progression in patients.

\section{DECLARATIONS}

\section{Authors' contributions}

All authors contributed equally to the article.

\section{Availability of data and materials}

Not applicable.

\section{Financial support and sponsorship}

This work was supported by Russian Science Foundation (18-15-00254).

\section{Conflicts of interest}

All authors declared that there are no conflicts of interest.

\section{Ethical approval and consent to participate}

Not applicable.

\section{Consent for publication}

Not applicable.

\section{Copyright}

(c) The Author(s) 2019.

\section{REFERENCES}

1. Sobenin IA, Galitsyna EV, Grechko AV, Orekhov AN. Small dense and desialylated low density lipoprotein in diabetic patients. Vessel Plus 2017;1:29-37.

2. Alipov VI, Sukhorukov VN, Karagodin VP, Grechko AV, Orekhov AN. Chemical composition of circulating native and desialylated low density lipoprotein: what is the difference? Vessel Plus 2017;1:107-15.

3. Takahashi S. Triglyceride rich lipoprotein -LPL-VLDL receptor and Lp(a)-VLDL receptor pathways for macrophage foam cell formation. J Atheroscler Thromb 2017;24:552-9.

4. Lehti S, Nguyen SD, Belevich I, Vihinen H, Heikkilä HM, et al. Extracellular lipids accumulate in human carotid arteries as distinct three-dimensional structures and have proinflammatory properties. Am J Pathol 2018;188:525-38.

5. Orekhov AN, Ivanova EA, Bobryshev YV. Naturally occurring multiple-modified low-density lipoprotein. In: Ruiz M, editor. Blood lipids and lipoproteins. New York: Nova Science Publishers Inc.; 2015. pp. 13-54.

6. Allahverdian S, Chaabane C, Boukais K, Francis GA, Bochaton-Piallat ML. Smooth muscle cell fate and plasticity in atherosclerosis. Cardiovasc Res 2018;114:540-50.

7. Kruth HS. Receptor-independent fluid-phase pinocytosis mechanisms for induction of foam cell formation with native low-density lipoprotein particles. Curr Opin Lipidol 2011;22:386-93.

8. Kruth HS, Jones NL, Huang W, Zhao B, Ishii I, et al. Macropinocytosis is the endocytic pathway that mediates macrophage foam cell formation with native low density lipoprotein. J Biol Chem 2005;280:2352-60.

9. Orekhov AN, Ivanova EA, Melnichenko AA, Sobenin IA. Circulating desialylated low density lipoprotein. Cor Vasa 2017;59:e149-56.

10. Orekhov AN, Sobenin IA. Modified lipoproteins as biomarkers of atherosclerosis. Front Biosci (Landmark Ed) 2018;23:1422-44.

11. Orekhov AN, Tertov VV, Mukhin DN, Koteliansky VE, Glukhova MA, et al. Association of low-density lipoprotein with particulate connective tissue matrix components enhances cholesterol accumulation in cultured subendothelial cells of human aorta. Biochim 
Biophys Acta 1987;928:251-8.

12. Virella G, Wilson K, Elkes J, Hammad SM, Rajab HA, et al. Immune complexes containing malondialdehyde (MDA) LDL induce apoptosis in human macrophages. Clin Immunol 2018;187:1-9.

13. Sánchez-Quesada JL, Villegas S, Ordóñez-Llanos J. Electronegative low-density lipoprotein. A link between apolipoprotein B misfolding, lipoprotein aggregation and proteoglycan binding. Curr Opin Lipidol 2012;23:479-86.

14. Orekhov AN, Andreeva ER, Bobryshev YV. Cellular mechanisms of human atherosclerosis: role of cell-to-cell communications in subendothelial cell functions. Tissue Cell 2016;48:25-34.

15. Orekhov AN, Oishi Y, Nikiforov NG, Zhelankin AV, Dubrovsky L, et al. Modified LDL particles activate inflammatory pathways in monocyte-derived macrophages: transcriptome analysis. Curr Pharm Des 2018;24:3143-51.

16. Nègre-Salvayre A, Augé N, Camaré C, Bacchetti T, Ferretti G, et al. Dual signaling evoked by oxidized LDLs in vascular cells. Free Radic Biol Med 2017;106:118-33.

17. Jongstra-Bilen J, Zhang CX, Wisnicki T, Li MK, White-Alfred S, et al. Oxidized low-density lipoprotein loading of macrophages downregulates TLR-Induced proinflammatory responses in a gene-specific and temporal manner through transcriptional control. J Immunol 2017;199:2149-57.

18. de la Paz Sánchez-Martínez M, Blanco-Favela F, Mora-Ruiz MD, Chávez-Rueda AK, Bernabe-García M, et al. IL-17-differentiated macrophages secrete pro-inflammatory cytokines in response to oxidized low-density lipoprotein. Lipids Health Dis 2017;16:196.

19. Orekhov AN, Ivanova EA. Cellular models of atherosclerosis and their implication for testing natural substances with antiatherosclerotic potential. Phytomedicine 2016;23:1190-7.

20. Singh RK, Haka AS, Brumfield A, Grosheva I, Bhardwaj P, et al. Ceramide activation of RhoA/Rho kinase impairs actin polymerization during aggregated LDL catabolism. J Lipid Res 2017;58:1977-87.

21. Guo K, Hu L, Xi D, Zhao J, Liu J, et al. PSRC1 overexpression attenuates atherosclerosis progression in apoE(-/-) mice by modulating cholesterol transportation and inflammation. J Mol Cell Cardiol 2018;116:69-80.

22. Liu XX, Zhang XW, Wang K, Wang XY, Ma WL, et al. Kuwanon G attenuates atherosclerosis by upregulation of LXR $\alpha$-ABCA1/ ABCG1 and inhibition of NFKB activity in macrophages. Toxicol Appl Pharmacol 2018;341:56-63.

23. Ooi BK, Goh BH, Yap WH. Oxidative stress in cardiovascular diseases: involvement of Nrf2 antioxidant redox signaling in macrophage foam cells formation. Int J Mol Sci 2017; doi: 10.3390/ijms18112336.

24. Yang Y, Li X, Peng L, An L, Sun N, et al. Tanshindiol C inhibits oxidized low-density lipoprotein induced macrophage foam cell formation via a peroxiredoxin 1 dependent pathway. Biochim Biophys Acta Mol Basis Dis 2018;1864:882-90.

25. Liu Z, Zhu H, Dai X, Wang C, Ding Y, et al. Macrophage liver kinase B1 inhibits foam cell formation and atherosclerosis. Circ Res 2017;121:1047-57.

26. Srikakulapu P, Upadhye A, Rosenfeld SM, Marshall MA, McSkimming C, et al. Perivascular adipose tissue harbors atheroprotective IgM-producing B cells. Front Physiol 2017;8:719.

27. Srikakulapu P, McNamara CA. B cells and atherosclerosis. Am J Physiol Heart Circ Physiol 2017;312:H1060-7.

28. Yan D, He Y, Dai J, Yang L, Wang X, et al. Vascular endothelial growth factor modified macrophages transdifferentiate into endotheliallike cells and decrease foam cell formation. Biosci Rep 2017; doi: 10.1042/BSR20170002.

29. Maier A, Wu H, Cordasic N, Oefner P, Dietel B, et al. Hypoxia-inducible protein 2 Hig2/Hilpda mediates neutral lipid accumulation in macrophages and contributes to atherosclerosis in apolipoprotein E-deficient mice. FASEB J 2017;31:4971-84.

30. Chen L, Zhang J, Deng X, Liu Y, Yang X, et al. Lysophosphatidic acid directly induces macrophage-derived foam cell formation by blocking the expression of SRBI. Biochem Biophys Res Commun 2017;491:587-94.

31. Brotman DJ, Walker E, Lauer MS, O’Brien RG. In search of fewer independent risk factors. Arch Intern Med 2005;165:138-45.

32. Stone NJ, Robinson JG, Lichtenstein AH, Bairey Merz CN, Blum CB, et al. 2013 ACC/AHA guideline on the treatment of blood cholesterol to reduce atherosclerotic cardiovascular risk in adults: a report of the American College of Cardiology/American Heart Association Task Force on Practice Guidelines. Circulation 2014;129:S1-45.

33. Catapano AL, Graham I, De Backer G, Wiklund O, Chapman MJ, et al. 2016 ESC/EAS guidelines for the management of dyslipidaemias: the task force for the management of dyslipidaemias of the European Society of Cardiology (ESC) and European Atherosclerosis Society (EAS) developed with the special contribution of the European Association for Cardiovascular Prevention \& Rehabilitation (EACPR). Atherosclerosis 2016;253:281-344.

34. Graham IM, Catapano AL, Wong ND. Current guidelines on prevention with a focus on dyslipidemias. Cardiovasc Diagn Ther 2017;7(Suppl 1):S4-10.

35. Kooter AJ, Kostense PJ, Groenewold J, Thijs A, Sattar N, et al. Integrating information from novel risk factors with calculated risks: the critical impact of risk factor prevalence. Circulation 2011;124:741-5.

36. Ioannidis JP, Tzoulaki I. Minimal and null predictive effects for the most popular blood biomarkers of cardiovascular disease. Circ Res 2012;110:658-62.

37. Gilstrap LG, Wang TJ. Biomarkers and cardiovascular risk assessment for primary prevention: an update. Clin Chem 2012;58:72-82.

38. Tzoulaki I, Siontis KC, Evangelou E, Ioannidis JP. Bias in associations of emerging biomarkers with cardiovascular disease. JAMA Intern Med 2013;173:664-71.

39. Orekhov AN, Tertov VV, Kabakov AE, Adamova IYu, Pokrovsky SN, et al. Autoantibodies against modified low density lipoprotein. Nonlipid factor of blood plasma that stimulates foam cell formation. Arterioscler Thromb 1991;11:316-26.

40. Tsimikas S, Miller YI. Oxidative modification of lipoproteins: mechanisms, role in inflammation and potential clinical applications in 
cardiovascular disease. Curr Pharm Des 2011;17:27-37.

41. Ivanova EA, Bobryshev YV, Orekhov AN. LDL electronegativity index: a potential novel index for predicting cardiovascular disease. Vasc Health Risk Manag 2015;11:525-32.

42. Akyol S, Lu J, Akyol O, Akcay F, Armutcu F, et al. The role of electronegative low-density lipoprotein in cardiovascular diseases and its therapeutic implications. Trends Cardiovasc Med 2017;27:239-46.

43. Sobenin IA, Salonen JT, Zhelankin AV, Melnichenko AA, Kaikkonen J, et al. Low density lipoprotein-containing circulating immune complexes: role in atherosclerosis and diagnostic value. Biomed Res Int 2014;2014:205697.

44. Sobenin IA, Karagodin VP, Melnichenko AC, Bobryshev YV, Orekhov AN. Diagnostic and prognostic value of low density lipoproteincontaining circulating immune complexes in atherosclerosis. J Clin Immunol 2013;33:489-95.

45. Orekhov AN, Kalenich OS, Tertov VV, Novikov ID. Lipoprotein immune complexes as markers of atherosclerosis. Int J Tissue React 1991;13:233-6.

46. Lopes-Virella MF, McHenry MB, Lipsitz S, Yim E, Wilson PF, et al. Immune complexes containing modified lipoproteins are related to the progression of internal carotid intima-media thickness in patients with type 1 diabetes. Atherosclerosis 2007;190:359-69.

47. Lopes-Virella MF, Hunt KJ, Baker NL, Virella G; VADT Group of Investigators. High levels of AGE-LDL, and of IgG antibodies reacting with MDA-lysine epitopes expressed by oxLDL and MDA-LDL in circulating immune complexes predict macroalbuminuria in patients with type 2 diabetes. J Diabetes Complications 2016;30:693-9.

48. Orekhov AN, Bobryshev YV, Sobenin IA, Melnichenko AA, Chistiakov DA. Modified low density lipoprotein and lipoproteincontaining circulating immune complexes as diagnostic and prognostic biomarkers of atherosclerosis and type 1 diabetes macrovascular disease. Int J Mol Sci 2014;15:12807-41.

49. Sobenin IA, Orekhova VA, Melnichenko AA, Bobryshev YV, Orekhov AN. Low density lipoprotein-containing circulating immune complexes have better prognostic value in carotid intima-media thickness progression than other lipid parameters. Int J Cardiol 2013;166:747-8.

50. Leopold JA. Antioxidants and coronary artery disease: from pathophysiology to preventive therapy. Coron Artery Dis 2015;26:176-83.

51. American Heart Association Nutrition Committee, Lichtenstein AH, Appel LJ, Brands M, Carnethon M, et al. Diet and lifestyle recommendations revision 2006: a scientific statement from the American Heart Association Nutrition Committee. Circulation 2006;114:82-96.

52. Sampson UK, Fazio S, Linton MF. Residual cardiovascular risk despite optimal LDL cholesterol reduction with statins: the evidence, etiology, and therapeutic challenges. Curr Atheroscler Rep 2012;14:1-10.

53. Cholesterol Treatment Trialists' (CTT) Collaboration, Fulcher J, O'Connell R, Voysey M, Emberson J, et al. Efficacy and safety of LDL-lowering therapy among men and women: meta-analysis of individual data from 174,000 participants in 27 randomised trials. Lancet 2015;385:1397-405.

54. Long-Term Intervention with Pravastatin in Ischaemic Disease (LIPID) Study Group. Prevention of cardiovascular events and death with pravastatin in patients with coronary heart disease and a broad range of initial cholesterol levels. N Engl J Med 1998;339:1349-57.

55. Heart Protection Study Collaborative Group. MRC/BHF heart protection study of cholesterol lowering with simvastatin in 20,536 highrisk individuals: a randomised placebo-controlled trial. Lancet 2002;360:7-22.

56. Downs JR, Clearfield M, Weis S, Whitney E, Shapiro DR, et al. Primary prevention of acute coronary events with lovastatin in men and women with average cholesterol levels: results of AFCAPS/TexCAPS. Air Force/Texas Coronary Atherosclerosis Prevention Study. JAMA 1998;279:1615-22.

57. Sacks FM, Pfeffer MA, Moye LA, Rouleau JL, Rutherford JD, et al. The effect of pravastatin on coronary events after myocardial infarction in patients with average cholesterol levels. Cholesterol and recurrent events trial investigators. N Engl J Med 1996;335:1001-9.

58. Shepherd J, Cobbe SM, Ford I, Isles CG, Lorimer AR, et al. Prevention of coronary heart disease with pravastatin in men with hypercholesterolemia. West of Scotland Coronary Prevention Study Group. N Engl J Med 1995;333:1301-7.

59. Cholesterol Treatment Trialists' (CTT) Collaborators. Efficacy and safety of cholesterol-lowering treatment: prospective meta-analysis of data from 90,056 participants in 14 randomised trials of statins. Lancet 2005;366:1267-78.

60. Chen Z, Peto R, Collins R, MacMahon S, Lu J, et al. Serum cholesterol concentration and coronary heart disease in population with low cholesterol concentrations. BMJ 1991;303:276-82.

61. Sacks FM, Tonkin AM, Shepherd J, Braunwald E, Cobbe S, et al. Effect of pravastatin on coronary disease events in subgroups defined by coronary risk factors: the Prospective Pravastatin Pooling Project. Circulation 2000;102:1893-900.

62. Kijani S, Vázquez AM, Levin M, Borén J, Fogelstrand P. Intimal hyperplasia induced by vascular intervention causes lipoprotein retention and accelerated atherosclerosis. Physiol Rep 2017; doi: 10.14814/phy2.13334.

63. Borén J, Williams KJ. The central role of arterial retention of cholesterol-rich apolipoprotein-B-containing lipoproteins in the pathogenesis of atherosclerosis: a triumph of simplicity. Curr Opin Lipidol 2016;27:473-83.

64. Chistiakov DA, Myasoedova VA, Revin VV, Orekhov AN, Bobryshev YV. The phenomenon of atherosclerosis reversal and regression: lessons from animal models. Exp Mol Pathol 2017;102:138-45.

65. Orekhov AN. Direct anti-atherosclerotic therapy; development of natural anti-atherosclerotic drugs preventing cellular cholesterol retention. Curr Pharm Des 2013;19:5909-28.

66. Zakiev ER, Nikiforov NG, Orekhov AN. Cell-based models for development of antiatherosclerotic therapies. Biomed Res Int 2017;2017:5198723.

67. Orekhov AN, Tertov VV, Novikov ID, Krushinsky AV, Andreeva ER, et al. Lipids in cells of atherosclerotic and uninvolved human 
aorta. I. Lipid composition of aortic tissue and enzyme isolated and cultured cells. Exp Mol Pathol 1985;42:117-37.

68. Orekhov AN, Tertov VV, Kudryashov SA, Khashimov KhA, Smirnov VN. Primary culture of human aortic intima cells as a model for testing antiatherosclerotic drugs. Effects of cyclic AMP, prostaglandins, calcium antagonists, antioxidants, and lipid-lowering agents. Atherosclerosis 1986;60:101-10.

69. Orekhov AN, Sobenin IA, Korneev NV, Kirichenko TV, Myasoedova VA, et al. Anti-atherosclerotic therapy based on botanicals. Recent Pat Cardiovasc Drug Discov 2013;8:56-66.

70. Kirichenko TV, Sobenin IA, Nikolic D, Rizzo M, Orekhov AN. Anti-cytokine therapy for prevention of atherosclerosis. Phytomedicine 2016;23:1198-210.

71. Kirichenko TV, Myasoedova VA, Orekhova VA, Ravani AL, Nikitina NA, et al. Phytoestrogen-rich natural preparation for treatment of climacteric syndrome and atherosclerosis prevention in perimenopausal women. Phytother Res 2017;31:1209-14.

72. Slevin M, Ahmed N, Wang Q, McDowell G, Badimon L. Unique vascular protective properties of natural products: supplements or future main-line drugs with significant anti-atherosclerotic potential? Vasc Cell 2012;4:9.

73. Orekhov AN, Sobenin IA. Modified and dysfunctional lipoproteins in atherosclerosis: effectors or biomarkers? Curr Med Chem 2018; doi: 10.2174/0929867325666180320121137. 